\title{
What about cardiovascular toxicities of immune checkpoint inhibitors?
}

\author{
Raquel Moreno Diaz (D) ,' Mario Cazalla Garcia, ${ }^{1}$ \\ Maria Pilar Bautista Sanz, ${ }^{1}$ Rosa Gonzalez Davia, ${ }^{2}$ \\ Adriana Carolina Rosero Rodriguez ${ }^{3}$
}

Decades ago, cancer patients were treated in cardiology consultations due to unexpected adverse effects arising from the use of traditional chemotherapy that had not been anticipated. Recently, Ball et al reviewed the cardiotoxicity associated with treatments with immune checkpoint inhibitors (ICIs). Their work was published on 1 October 2019 in the review topic of the week in the Journal of the American College of Cardiology. ${ }^{1}$ The publication described the cardiovascular adverse events associated with exposure to ICIs based on the literature of the last few years. The authors carried out a complete review, expanding the results and perspectives obtained from clinical trials to include the real-life results of patients.

Myocarditis is the most common adverse cardiovascular event associated with ICIs (45\%), with a rate of fulminant myocarditis of $15 \%$. The mortality rate is $50 \%$, while the mortality rate associated with pericardial disease or vasculitis is $21 \%$ and $6 \%$, respectively. Takosubo syndrome, arrhythmias and vasculitis are other important cardiovascular events. ${ }^{1}$ Despite being uncommon, they are also serious adverse events.

Most adverse events with ICIs occur within the first 4 months of treatment, but cardiovascular events can occur at any time during treatment or even a few months after discontinuation. The average time for the onset of myocarditis is $17-34$ days. However, it usually appears near the beginning of the administration of the first dose of ICI. In addition, studies based on some mouse species showed that the genetic deletion of PDL1/PDL2 as well as treatment with anti-PDL1 can transform transient myocarditis into a lethal disease. ${ }^{2}$

\footnotetext{
'Pharmacy Department, Hospital Universitario Infanta Cristina, Parla, Spain

${ }^{2}$ Cardiology, Hospital Universitario Infanta Cristina, Parla, Spain

${ }^{3}$ Oncology, Hospital Universitario Infanta Cristina, Parla, Spain
}

Correspondence to Raquel Moreno Diaz, Pharmacy, Hospital Universitario Infanta Cristina, Parla 28981, Spain; rmorenod@salud.madrid.org
The diagnosis of heart disease is reached after the finding of elevated cardiac biomarkers or the appearance of arrhythmia on an ECG. The clinical manifestation may be varied, with some patients remaining asymptomatic while others experience dyspnoea or even severe hypotension due to cardiogenic shock.

At first the pharmaceutical companies did not record the frequency of myocarditis in the safety database of their clinical trials. ${ }^{2}$ The fact that cardiac events appeared to have a low incidence led to an underestimation of cardiac toxicity in reports related to ICIs. There is an evident inconsistency of data summarised in clinical trial records regarding associated cardiovascular events; continuous cardiac monitoring (including assessment of troponin) was not included in most of the immunotherapy trial protocols. ${ }^{2}$

In the first publications most articles related to the toxicity of ICIs underestimated and even rejected possible toxicity at the cardiac level. ${ }^{2}$ Fortunately, the situation is changing and the number of scientific publications evaluating this topic is growing. For all the reasons mentioned above, cardiotoxicity has rarely been suspected as an adverse event related to this type of drug. However, the actual incidence of both early and delayed cardiotoxicity associated with the use of ICIs is still unknown.

In addition, it is necessary to take into account the characteristics of each patient. Chemotherapy-related toxicity has been shown to occur more frequently in elderly patients than in young patients. ${ }^{3}$ In clinical trials, the elderly population is not adequately represented ${ }^{3}$ and, consequently, it is not surprising that ICIs have not shown either efficacy or age-dependent toxicity in clinical trials. However, in real-life it has been seen that elderly and more vulnerable patients have a higher incidence of adverse events and tend to stop treatment earlier. ${ }^{3}$

The cardiotoxicity related to ICIs has been classified into four degrees based on the different symptoms of the patient, the level of elevation of the cardiac biomarkers and the echocardiographic findings. ${ }^{4}$ Permanent discontinuation should be considered for those who reach a higher risk category than grade 1 .

Following the article by Ball et al, we conducted an observational study in our hospital, including all patients undergoing treatment with ICIs during the year 2019. Forty patients treated with durvalumab, atezolizumab, nivolumab or pembrolizumab were included. Five of the patients suffered a cardiovascular event, of which two had a cardiac history in their medical history. Some of the events we found were retrocardiac pain, tachycardia, pericardial effusion and left ventricular dysfunction. A case of grade 2 ventricular dysfunction and pericardium effusion associated with the use of atezolizumab was reported. Although the patient required hospitalisation, treatment with atezolizumab was not interrupted and the ventricular ejection fraction improved after a few weeks. This patient had atrial fibrillation in his history that could have been decompensated; however, the involvement of atezolizumab could not be ruled out.

Based on all the information described, it seems that it is crucial to develop consensual guidelines for the management of the different forms of immunomediated cardiac toxicity. ${ }^{2}$ However, there are already some publications that should be read. ${ }^{1} 245$ Without this knowledge, optimal treatment cannot be guaranteed. In this sense, an interesting algorithm has been published to facilitate patient monitoring. ${ }^{1}$

Patients also have a lot to say and we have to encourage and empower them in their own care. They should be informed of how to recognise and to alert of any warning signs of cardiotoxicity such as dyspnoea, fatigue or chest pain. We therefore believe that we urgently need to have oncologists and cardiologists working together to detect and control the cardiotoxic effects of these new monoclonal antibodies. To achieve this, we need to identify all patients at risk of suffering an adverse cardiac event. We also recommend reviewing all the new information that is emerging, such as the analysis by Ball and colleagues.

We therefore emphasise the importance of collaboration between cardiologists and pharmacological oncologists to effectively recognise cardiovascular events in patients treated with ICIs to avoid a fatal outcome. 
Funding The authors have not declared a specific grant for this research from any funding agency in the public, commercial or not-for-profit sectors.

Competing interests None declared.

Patient consent for publication Not required.

Provenance and peer review Not commissioned; internally peer reviewed.

(c) European Association of Hospital Pharmacists 2021. No commercial re-use. See rights and permissions. Published by BMJ.

D) Check for updates
To cite Moreno Diaz R, Cazalla Garcia M, Bautista Sanz MP, et al. Eur J Hosp Pharm 2021;28:2-3.

Published Online First 23 April 2020

Eur J Hosp Pharm 2021;28:2-3.

doi:10.1136/ejhpharm-2020-002277

ORCID iD

Raquel Moreno Diaz http://orcid.org/0000-0002-81770351

\section{REFERENCES}

1 Ball S, Ghosh RK, Wongsaengsak S, et al. Cardiovascular toxicities of immune checkpoint inhibitors. J Am Coll Cardiol 2019;74:1714-27.
2 Varricchi G, Galdiero MR, Marone G, et al. Cardiotoxicity of immune checkpoint inhibitors. ESMO Open 2017;2:e000247.

3 van Holstein Y, Kapiteijn E, Bastiaannet E, et al. Efficacy and adverse events of immunotherapy with checkpoint inhibitors in older patients with cancer. Drugs Aging 2019;36:927-38.

4 UpToDate. Special considerations and toxicities associated with checkpoint inhibitor immunotherapy. Available: https://www.uptodate.com/

5 ESMO. What are immunotherapy side effects? Available: https://www.esmo.org/content/download/124130/ 2352601/file/ESMO-Patient-Guide-onlmmunotherapySide-Effects.pdf 